\title{
Drawing Diagrams with $\mathbf{R}$
}

by Paul Murrell

$\mathrm{R}$ provides a number of well-known high-level facilities for producing sophisticated statistical plots, including the "traditional" plots in the graphics package (R Development Core Team, 2008), the Trellisstyle plots provided by lattice (Sarkar, 2008), and the grammar-of-graphics-inspired approach of ggplot2 (Wickham, 2009).

However, $\mathrm{R}$ also provides a powerful set of lowlevel graphics facilities for drawing basic shapes and, more importantly, for arranging those shapes relative to each other, which can be used to draw a wide variety of graphical images. This article highlights some of R's low-level graphics facilities by demonstrating their use in the production of diagrams. In particular, the focus will be on some of the useful things that can be done with the low-level facilities provided by the grid graphics package (Murrell, 2002, 2005b,a).

\section{Starting at the end}

An example of the type of diagram that we are going to work towards is shown below. We have several "boxes" that describe table schema for a database, with lines and arrows between the boxes to show the relationships between tables.

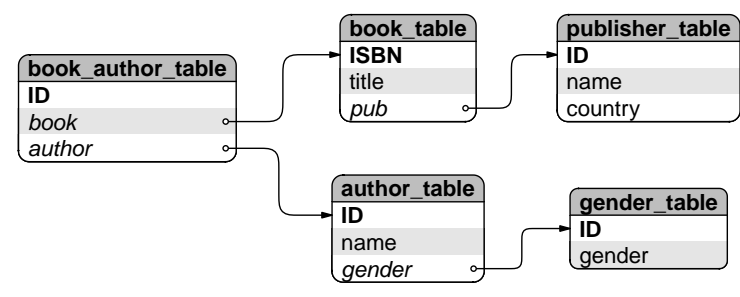

To forestall some possible misunderstandings, the sort of diagram that we are talking about is one that is designed by hand. This is not a diagram that has been automatically laid out.

The sort of diagram being addressed is one where the author of the diagram has a clear idea of what the end result will roughly look like- - the sort of diagram that can be sketched with pen and paper. The task is to produce a pre-planned design, using a computer to get a nice crisp result.

That being said, a reasonable question is "why not draw it by hand?", for example, using a freehand drawing program such as Dia (Larsson, 2008). The advantage of using $\mathrm{R}$ code to produce this sort of image is that code is easier to reproduce, reuse, maintain, and fine-tune with accuracy. The thought of creating this sort of diagram by pushing objects around the screen with a mouse fills me with dread. Maybe I'm just not a very GUI guy.

Before we look at drawing diagrams with the core $\mathrm{R}$ graphics facilties, it is important to acknowledge that several contributed $\mathrm{R}$ packages already provide facilities for drawing diagrams. The Rgraphviz (Gentry et al., 2008) and igraph (Csardi and Nepusz, 2006) packages provide automated layout of nodeand-edge graphs, and the shape and diagram packages (Soetaert, 2008b,a) provide functions for drawing nodes of various shapes with lines and arrows between them, with manual control over layout.

In this article, we will only be concerned with drawing diagrams with a small number of elements, so we do not need the automated layout facilities of Rgraphviz or igraph. Furthermore, while the shape and diagram packages provide flexible tools for building node-and-edge diagrams, the point of this article is to demonstrate low-level grid functions. We will use a node-and-edge diagram as the motivation, but the underlying ideas can be applied to a much wider range of applications.

In each of the following sections, we will meet a basic low-level graphical tool and demonstrate how it can be used in the generation of the pieces of an overall diagram, or how the tool can be used to combine pieces together in convenient ways.

\section{Graphical primitives}

One of the core low-level facilities of R graphics is the ability to draw basic shapes. The typical graphical primitives such as text, circles, lines, and rectangles are all available.

In this case, the shape of each box in our diagram is not quite as simple as a rectangle because it has rounded corners. However, a rounded rectangle is also one of the graphical primitives that the grid package provides. ${ }^{1}$

The code below draws a rounded rectangle with a text label in the middle.

$>$ library (grid)

$>$ grid.roundrect $($ width $=.25)$
$>$ grid.text ("ISBN")

ISBN

\footnotetext{
${ }^{1}$ From $\mathrm{R}$ version 2.9.0; prior to that, a simpler rounded rectangle was available via the grid.roundRect () function in the RGraphics package.
} 


\section{Viewports}

A feature of the boxes in the diagram at the beginning of this article is that the text is carefully positioned relative to the rounded rectangle; the text is left-aligned within the rectangle. This careful positioning requires knowing where the left edge of the rectangle is on the page. Calculating those positions is annoyingly tricky and only becomes more annoying if at some later point the position of the box is adjusted and the positions of the text labels have to be calculated all over again.

Using a grid viewport makes this sort of positioning very simple. The basic idea is that we can create a viewport where the box is going to be drawn and then do all of our drawing within that viewport. Positioning text at the left edge of a viewport is very straightforward, and if we need to shift the box, we simply shift the viewport and the text automatically tags along for the ride. All of this applies equally to positioning the text vertically within the box.

In the code below, we create a viewport for the overall box, we draw a rounded rectangle occupying the entire viewport, then we draw text $2 \mathrm{~mm}$ from the left hand edge of the viewport and 1.5 lines of text up from the bottom of the viewport. A second line of text is also added, 0.5 lines of text from the bottom.
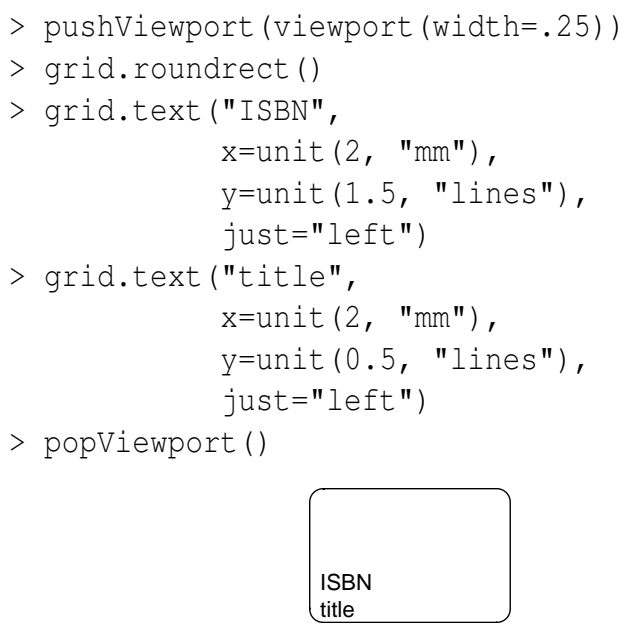

\section{Coordinate systems}

The positioning of the labels within the viewport in the previous example demonstrates another useful feature of the grid graphics system: the fact that locations can be specified in a variety of coordinate systems or units. In that example, the text was positioned horizontally in terms of millimetres and vertically in terms of lines of text (which is based on the font size in use).

As another example of the use of these different units, we can size the overall viewport so that it is just the right size to fit the text labels. In the following code, the height of the viewport is based on the number of labels and the width of the viewport is based on the width of the largest label, plus a $2 \mathrm{~mm}$ gap either side. This code also simplifies the labelling by drawing both labels in a single grid.text () call.

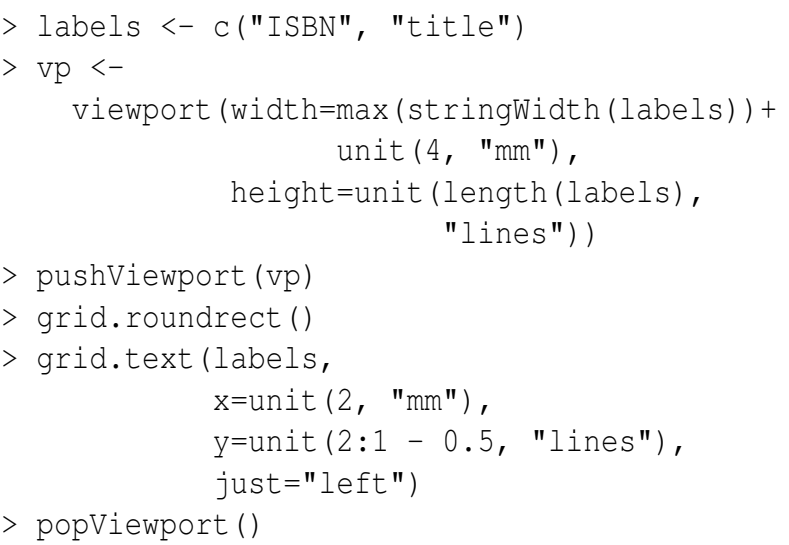

\section{Clipping}

Another feature of the boxes that we want to produce is that they have shaded backgrounds. Looking closely, there are some relatively complex shapes involved in this shading. For example, the grey background for the "heading" of each box has a curvy top, but a flat bottom. These are not simple rounded rectangles, but some unholy alliance of a rounded rectangle and a normal rectangle.

It is possible, in theory, to achieve any sort of shape with $\mathrm{R}$ because there is a general polygon graphical primitive. However, as with the positioning of the text labels, determining the exact boundary of this polygon is not trivial and there are easier ways to work.

In this case, we can achieve the result we want using clipping, so that any drawing that we do is only visible on a restricted portion of the page. $R$ does not provide clipping to arbitrary regions, but it is possible to set the clipping region to any rectangular region.

The basic idea is that we will draw the complete rounded rectangle, then set the clipping region for the box viewport so that no drawing can occur in the last line of text in the box and then draw the rounded rectangle again, this time with a different background. If we continue doing this, we end up with bands of different shading.

The following code creates an overall viewport for a box and draws a rounded rectangle with a grey fill. The code then sets the clipping region to start one line of text above the bottom of the viewport and draws another rounded rectangle with a white fill. The effect is to leave just the last line of the original 
grey rounded rectangle showing beneath the white rounded rectangle that has had its last line clipped.
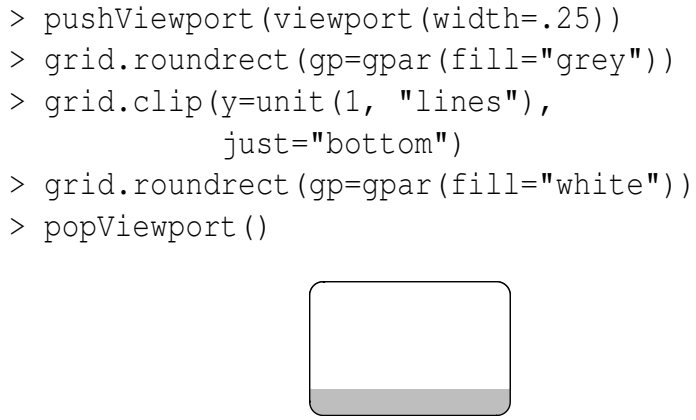

\section{Drawing curves}

Another basic shape that is used in the overall diagram is a nice curve from one box to another.

In addition to the basic functions to draw straight lines in $\mathrm{R}$, there are functions that draw curves. In particular, $R$ provides a graphical primitive called an $X$-spline (Blanc and Schlick, 1995). The idea of an Xspline is that we define a set of control points and a curve is drawn either through or near to the control points. Each control point has a parameter that specifies whether to create a sharp corner at the control point, or draw a smooth curve through the control point, or draw a smooth curve that passes nearby.

The following code sets up sets of three control points and draws an $\mathrm{X}$-spline relative to each set of control points. The first curve makes a sharp corner at the middle control point, the second curve makes a smooth corner through the middle control point, and the third curve makes a smooth corner near the middle control point. The control points are drawn as grey dots for reference (code not shown).
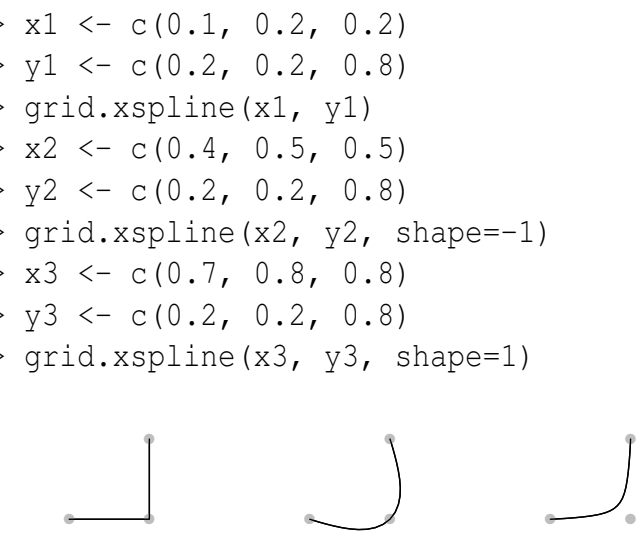

Determining where to place the control points for a curve between two boxes is another one of those annoying calculations, so a more convenient option is provided by a curve graphical primitive in grid. The idea of this primitive is that we simply specify the start and end points of the curve and R figures out a set of reasonable control points to produce an appropriate $\mathrm{X}$-spline. It is also straightforward to add an arrow to either end of any straight or curvy line that $\mathrm{R}$ draws.

The following code draws three curves between pairs of end points. The first curve draws the default "city-block" line between end points, with a smooth corner at the turning point, the second curve is similar, but with an extra corner added, and the third curve draws a single wide, smooth corner that is distorted towards the end point. The third curve also has an arrow at the end.
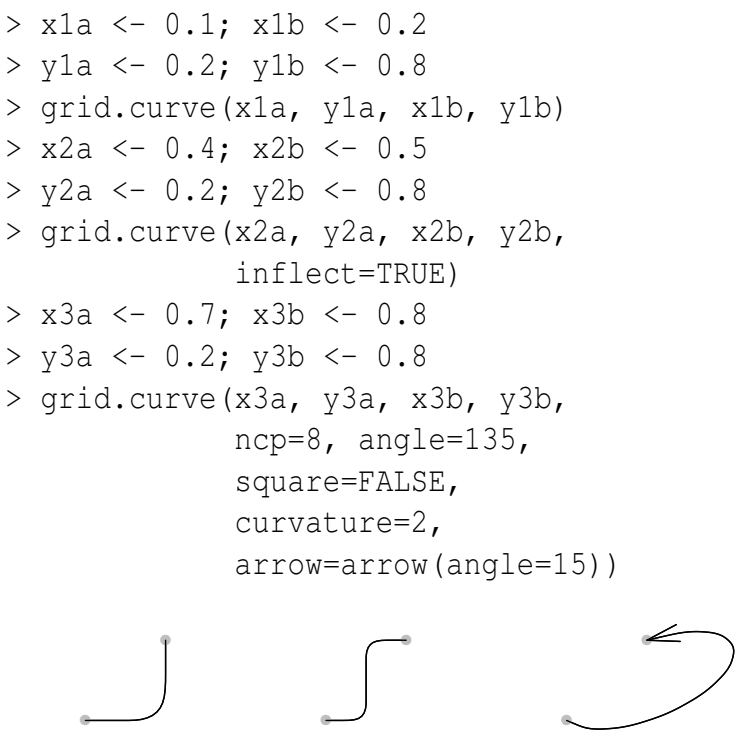

\section{Graphical functions}

The use of graphical primitives, viewports, coordinate systems, and clipping, as described so far, can be used to produce a box of the style shown in the diagram at the start of the article. For example, the following code produces a box containing three labels, with background shading to assist in differentiating among the labels.

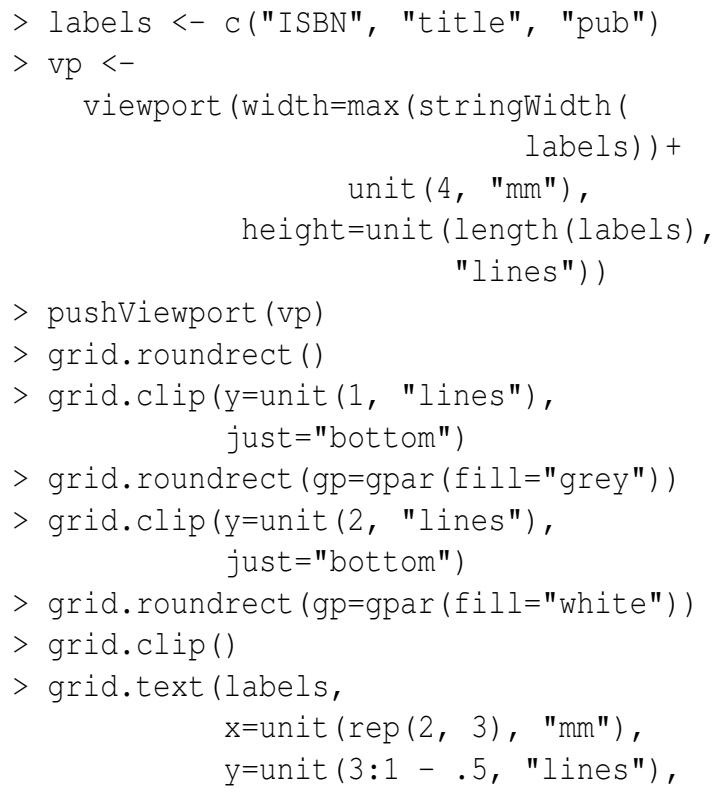




\section{just="left " ) \\ $>$ popViewport ()

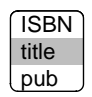

However, in the sort of diagram that we want to produce, there will be several such boxes. Rather than write separate code for each box, it makes sense to write a general function that will work for any set of labels. Such a function is shown in Figure 1 and the code below uses this function to draw two boxes side by side.

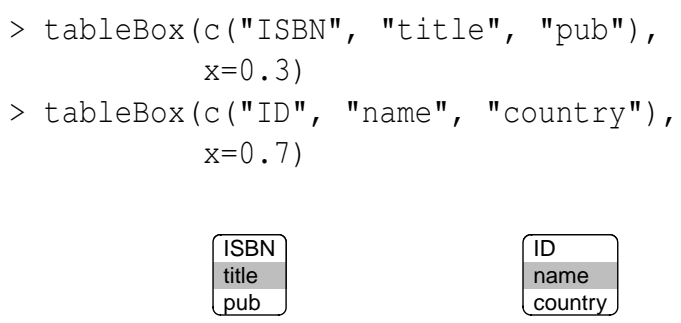

\begin{tabular}{|l|l|}
\hline $\begin{array}{l}\text { ISBN } \\
\text { title } \\
\text { pub }\end{array}$ & $\begin{array}{l}\text { ID } \\
\text { name } \\
\text { country }\end{array}$ \\
\hline
\end{tabular}

This function represents the simplest way to efficiently reuse graphics code and to provide graphics code for others to use. However, there are benefits to be gained from going beyond this procedural programming style to a slightly more complicated object-oriented approach.

\section{Graphical objects}

In order to achieve the complete diagram introduced at the start of this article, we need one more step: we need to draw lines and arrows from one box to another. We already know how to draw lines and curves between two points; the main difficulty that remains is calculating the exact position of the start and end points, because these locations depend on the locations and dimensions of the boxes. The calculations could be done by hand for each individual curve, but as we have seen before, there are easier ways to work. The crucial idea for this step is that we want to create not just a graphical function that encapsulates how to draw a box, but define a graphical object that encapsulates information about a box.

The code in Figure 2 defines such a graphical object, plus a few other things that we will get to shortly. The first thing to concentrate on is the boxGrob() function. This function creates a "box" graphical object. In order to do this, all it has to do is call the grob () function and supply all of the information that we want to record about "box" objects. In this case, we just record the labels to be drawn within the box and the location where we want to draw the box.

This function does not draw anything. For example, the following code creates two "box" objects, but produces no graphical output whatsoever.

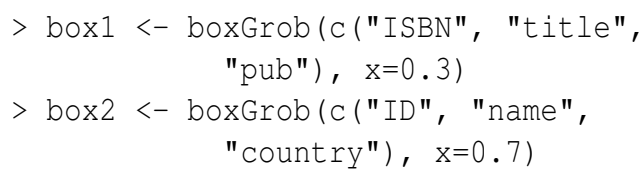

The grid.draw () function can be used to draw any graphical object, but we need to supply the details of how "box" objects get drawn. This is the purpose of the second function in Figure 2. This function is a method for the drawDetails () function; it says how to draw "box" objects. In this case, the function is very simple because it can call the tableBox () function that we defined in Figure 1. The important detail is that the boxGrob () function specified a special class, $\mathrm{cl}=$ "box", for "box" objects, which meant that we could define a drawDetails () method specifically for this sort of object and control what gets drawn.

With this drawDetails() method defined, we can draw the boxes that we created earlier by calling the grid.draw() function. This function will draw any grid graphical object by calling the appropriate method for the drawDetails () generic function (among other things). The following code calls grid.draw () to draw the two boxes.
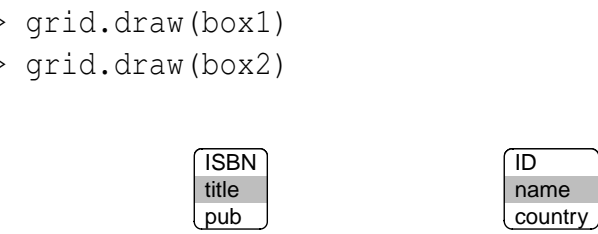

At this point, we appear to have achieved only a more complicated equivalent of the previous graphics function. However, there are a number of other functions that can do useful things with grid graphical objects. For example, the grobX() and groby () functions can be used to calculate locations on the boundary of a graphical object. As with grid.draw (), which has to call drawDetails () to find out how to draw a particular class of graphical object, these functions call generic functions to find out how to calculate locations on the boundary for a particular class of object. The generic functions are called xDetails() and yDetails() and methods for our special "box" class are defined in the last two functions in Figure 2.

These methods work by passing the buck. They both create a rounded rectangle at the correct location and the right size for the box, then call grobX () (or groby ()) to determine a location on the boundary of the rounded rectangle. In other words, they rely on code within the grid package that already exists to calculate the boundary of rounded rectangles.

With these methods defined, we are now in a position to draw a curved line between our boxes. The key idea is that we can use grobX () and groby () to specify a start and end point for the curve. For example, we can start the curve at the right hand edge of 


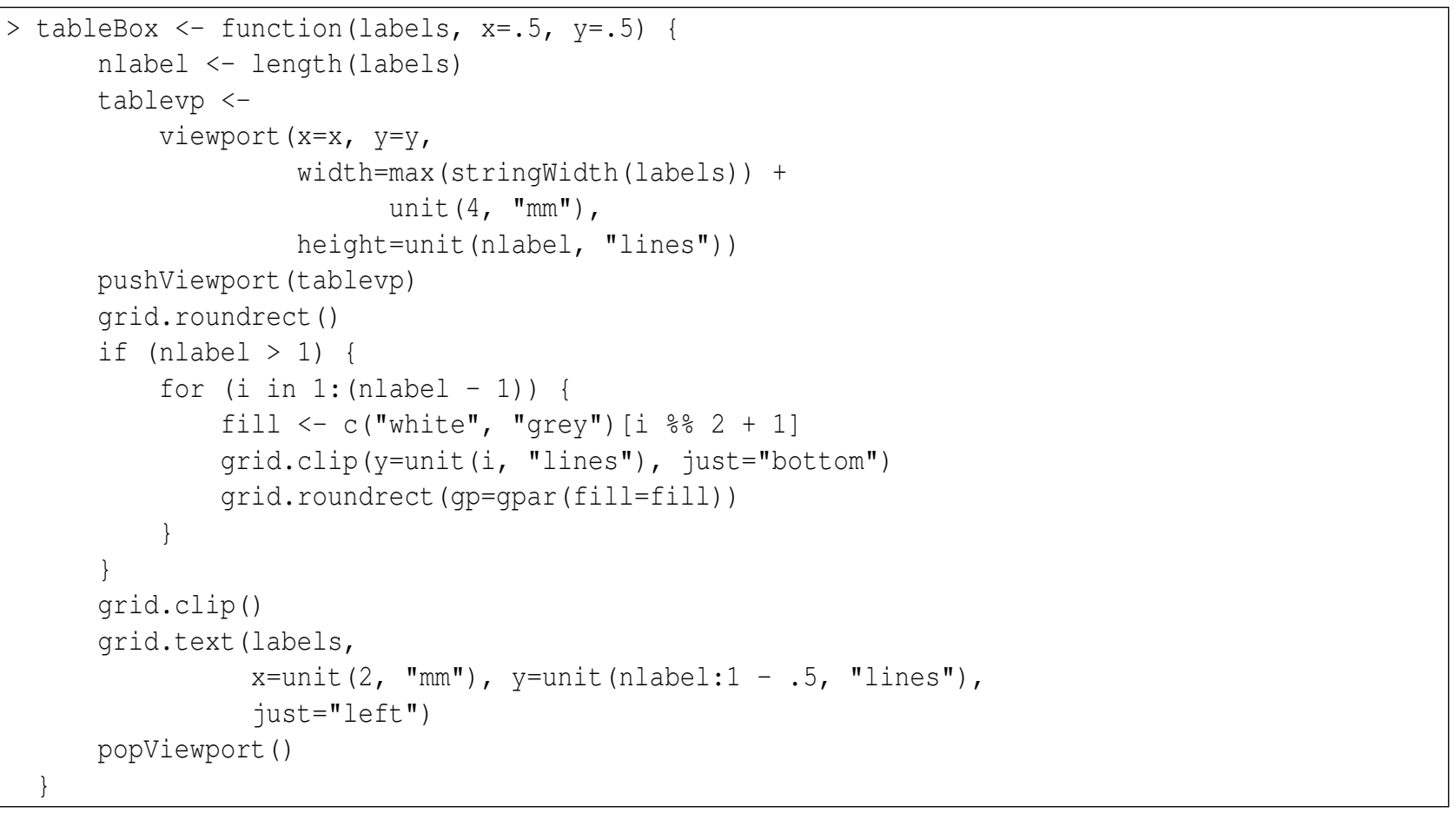

Figure 1: A function to draw a diagram box, for a given set of labels, centred at the specified (x, y) location.

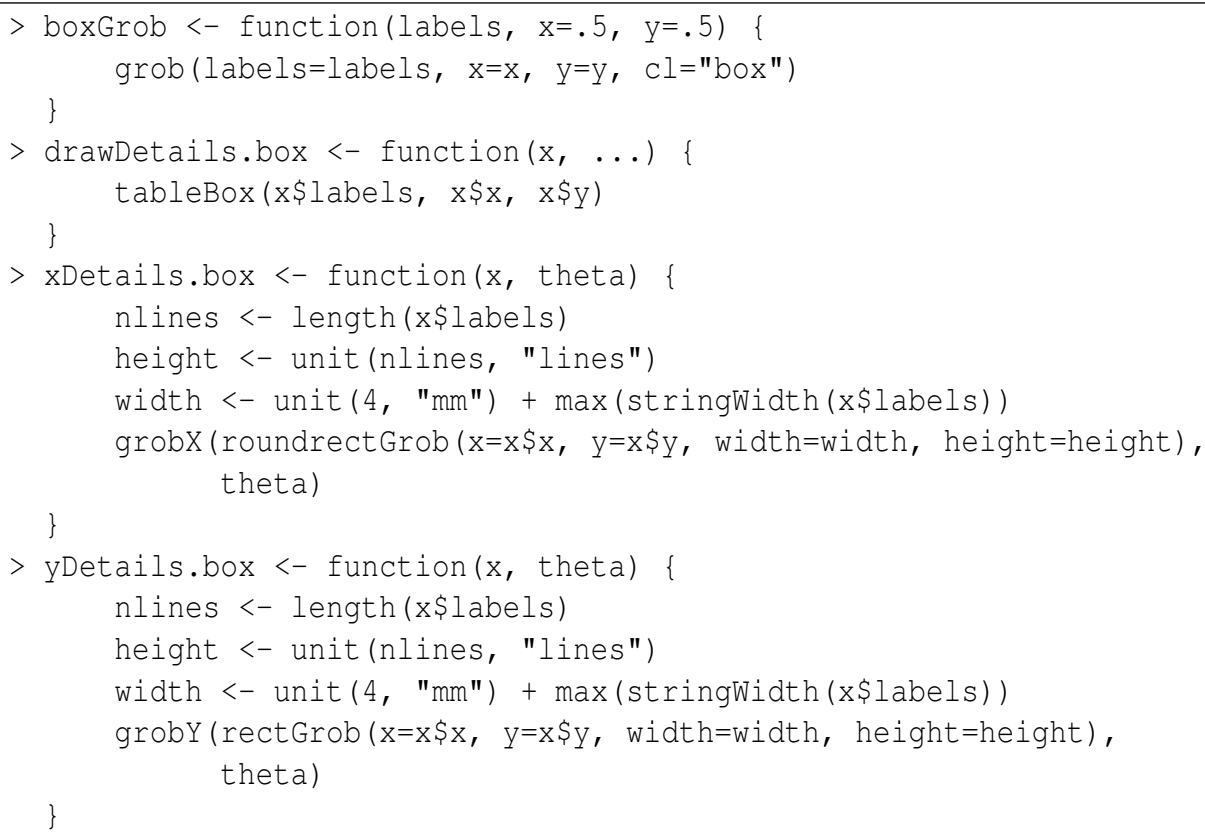

Figure 2: Some functions that define a graphical object representing a diagram box. The boxGrob () function constructs a "box" object, the drawDetails () method describes how to draw a "box" object, and the xDetails () and yDetails () functions calculate locations on the boundary of a "box" object. 
box1 by specifying grobx (box1, "east"). The vertical position is slightly trickier because we do not want the line starting at the top or bottom of the box, but we can simply add or subtract the appropriate number of lines of text to get the right spot.

The following code uses these ideas to draw a curve from the pub label of box 1 to the ID label of box2. The curve has two corners (inflect=TRUE) and it has a small arrow at the end.

This call to grid.curve() is relatively verbose, but in a diagram containing many similar curves, this burden can be significantly reduced by writing a simple function that hides away the common features, such as the specification of the arrow head.

The major gain from this object-oriented approach is that the start and end points of this curve are described by simple expressions that will automatically update if the locations of the boxes are modified.

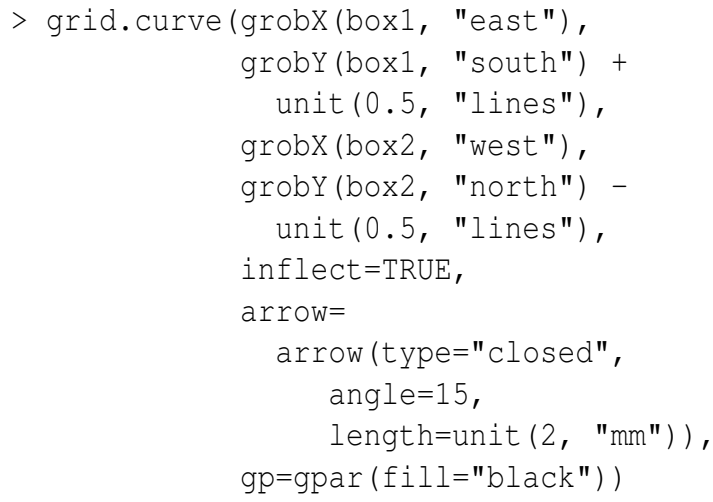

\section{Conclusion}

This article has demonstrated a number of useful low-level graphical facilities in $\mathrm{R}$ with an example of how they can be combined to produce a diagram consisting of non-trivial nodes with smooth curves between them.

The code examples provided in this article have ignored some details in order to keep things simple. For example, there are no checks that the arguments have sensible values in the functions tableBox () and boxGrob (). However, for creating one-off diagrams, this level of detail is not necessary anyway.

One detail that would be encountered quite quickly in practice, in this particular sort of diagram, is that a curve from one box to another that needs to go across-and-down rather than across-andup would require the addition of curvature $=-1$ to the grid.curve() call. Another thing that is missing is complete code to produce the example diagram from the beginning of this article, where there are five interconnected boxes and the boxes have some additional features, such as a distinct "header" line at the top. This complete code was excluded to save on space, but a simple $\mathrm{R}$ package is provided at http://www.stat.auckland.ac.nz/ paul/ with code to draw that complete diagram and the package also contains a more complete implementation of code to create and draw "box" graphical objects.

One final point is that using $\mathrm{R}$ graphics to draw diagrams like this is not fast. In keeping with the $S$ tradition, the emphasis is on developing code quickly and on having code that is not a complete nightmare to maintain. In this case particularly, the speed of developing a diagram comes at the expense of the time taken to draw the diagram. For small, one-off diagrams this is not likely to be an issue, but the approach described in this article would not be appropriate, for example, for drawing a node-and-edge graph of the Internet.

\section{Bibliography}

C. Blanc and C. Schlick. X-splines: a spline model designed for the end-user. In SIGGRAPH'95: Proceedings of the 22nd annual conference on Computer graphics and interactive techniques, pages 377-386, New York, NY, USA, 1995. ACM. ISBN 0-89791-701-4. doi: http:/ / doi.acm.org/10.1145/218380.218488.

G. Csardi and T. Nepusz. The igraph software package for complex network research. InterJournal, Complex Systems:1695, 2006. URL http:// igraph.sf.net.

J. Gentry, L. Long, R. Gentleman, S. Falcon, F. Hahne, and D. Sarkar. Rgraphviz: Provides plotting capabilities for $R$ graph objects, 2008 . R package version 1.18.1.

A. Larsson. Dia, 2008. http://www.gnome.org/ projects/dia/.

P. Murrell. The grid graphics package. R News, 2(2): 14-19, June 2002. URL http://CRAN.R-project. org/doc/Rnews/.

P. Murrell. Recent changes in grid graphics. $R$ News, 5(1):12-20, May 2005a. URL http: / / CRAN. R-project.org/doc/Rnews/.

P. Murrell. $R$ Graphics. Chapman \& Hall/CRC, 2005b. URL http://www.stat.auckland.ac.nz/ paul/RGraphics/rgraphics.html. ISBN 1-58488486-X.

R Development Core Team. R: A Language and Environment for Statistical Computing. R Foundation for Statistical Computing, Vienna, Austria, 2008. URL http: / / www. R-project . org. ISBN 3-900051-07-0. 
D. Sarkar. Lattice: Multivariate Data Visualization with R. Springer, 2008. URL http://amazon.com/o/ ASIN/0387759689/. ISBN 9780387759685.

K. Soetaert. diagram: Functions for visualising simple graphs (networks), plotting flow diagrams, 2008a. R package version 1.2.

K. Soetaert. shape: Functions for plotting graphical shapes, colors, 2008b. R package version 1.2.
H. Wickham. ggplot2. Springer, 2009. http://had. co.nz/ggplot2/.

Paul Murrell

Department of Statistics

The University of Auckland

New Zealand

pauldstat.auckland.ac.nz 